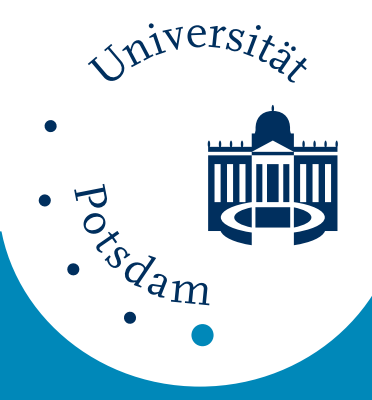

Universität Potsdam

Jürgen Kurths, A. Voss, Annette Witt, P. Saparin, H. J. Kleiner, N. Wessel

Quantitative analysis of heart rate variability

NLD Preprints ; 5 


\title{
Quantitative Analysis of Heart Rate Variability
}

\author{
J. Kurths ${ }^{1}$, A. Voss ${ }^{2}$, A. Witt ${ }^{1}$, \\ P. Saparin ${ }^{3}$, H. J. Kleiner ${ }^{2}$, N. Wessel ${ }^{2}$ \\ 1 Arbeitsgruppe Nichtlineare Dynamik der Max-Planck-Gesellschaft \\ an der Universität Potsdam, Pf. 601553, D-14415 Potsdam, Germany \\ ${ }^{2}$ MDC, Franz-Volhard-Klinik, Wiltbergstr. 50, D-13125 Buch, Germany \\ ${ }^{3}$ Saratov State University, Astrakhanskaja U1. 40, Russia
}

\begin{abstract}
In the modern industrialized countries every year several hundred thousands of people die due to the sudden cardiac death. The individual risk for this sudden cardiac death cannot be defined precisely by common available, non-invasive diagnostic tools like Holter-monitoring, highly amplified ECG and traditional linear analysis of heart rate variability (HRV). Therefore, we apply some rather unconventional methods of nonlinear dynamics to analyse the HRV. Especially, some complexity measures that are basing on symbolic dynamics as well as a new measure, the renormalized entropy, detect some abnormalities in the HRV of several patients who have been classified in the low risk group by traditional methods. A combination of these complexity measures with the parameters in the frequency domain seems to be a promising way to get a more precise definition of the individual risk. These findings have to be validated by a representative number of patients.
\end{abstract}




\section{Introduction}

Ventricular arrhythmia, especially ventricular tachycardia (VT) and ventricular fibrillations are in many cases the cause of sudden cardiac death of patients after myocardial infarction. The improved identification of patients highly threatened by these severe rhythm disturbances is an important and very actual clinical problem.

Short as well as long-range fluctuations in the heart rate are related to the autonomic nervous system control of heart activity and vasomotion. Recent studies have shown that a low heart rate variability (HRV) is a clear indication of an increased risk for severe ventricular arrhythmia and sudden cardiac death. These phenomena seem to be associated with a structural change of the beat to beat interval dynamics.

Kleiger [6] showed that a reduced HRV carries an adverse prognosis in patients who have survived an acute myocardial infarction. Malik [9] examined HRV in those patients to find the optimum time and duration of recording of the ambulatory ECG for the prediction of the risk of a sudden cardiac death, or serious arrhytmic events. It has been reported that patients after an acute myocardial infarction have a reduced parasympathetic function which causes an increased sympathetic tonus.

Figure 1: Scheme of different techniques for the analysis of ECG 
These techniques can be divided in time and frequency domain.

Therefore, several well-known techniques have been applied to detect such high risk patients from ECG (Fig. 1). Firstly, some rather simple time domain measures of heart rate variability have been proven useful for clinical purposes. Secondly, the spectral analysis of the RR time series that expresses HRV in the frequency domain exhibits different oscillating sources of the variability of heart beat generation. The different regions in the power spectrum are related to special physiological phenomena. We have considered the following: The frequency band $<0.0033$ $\mathrm{Hz}$ (ultra low frequancy power ULF) and the frequency band $0.0033 \ldots 0.05 \mathrm{~Hz}$ (very low frequency power VLF) represents humoral, vasomotion and thermo regulations and reflects also the activity of the renin-angiotensin-aldosteron system. The frequency band $0.05 \ldots 0.15 \mathrm{~Hz}$ (low frequency power LF) reflects modulation of sympathetic or parasympathetic tone by baroflex activity (blood pressure regulation) and the frequency band $0.15 \ldots 0.45 \mathrm{~Hz}$ (high frequency power HF) represents the modulation of vagal activity especially influenced by respiration. Bigger [1] showed that the day-to-day stability of the measure of heart period variability makes it possible to detect small changes due to progression, regression of diseases or treatment effects. Further on he pointed out that according to Kleigers results especially the ULF of the spectrum has the strongest association with mortality in post infarction patients.

However, the traditional techniques of data analysis in time and frequency domain are often not sufficient to characterize the complex dynamics of heart beat generation. Hence, different attempts have been reported to apply the concept of nonlinear dynamics to this problem [3]. After some optimism in the 80-ies, it has become clear that the HRV cannot be generally characterized by low fractal dimensions.

The purpose of this contribution is, therefore, to analyze the HRV by means of other methods of nonlinear dynamics which are based on the concept of symbolic dynamics and on a renormalized entropy.

The organization of this paper is as follows: The kind of the data and the traditional techniques to analyze them are described in section 2 . In section 3 we introduce different complexity measures. Their efficiency to detect high risk patients is discussed in section 4 which also includes a comparison with the results obtained from traditional techniques. Section 5 concludes the paper. 

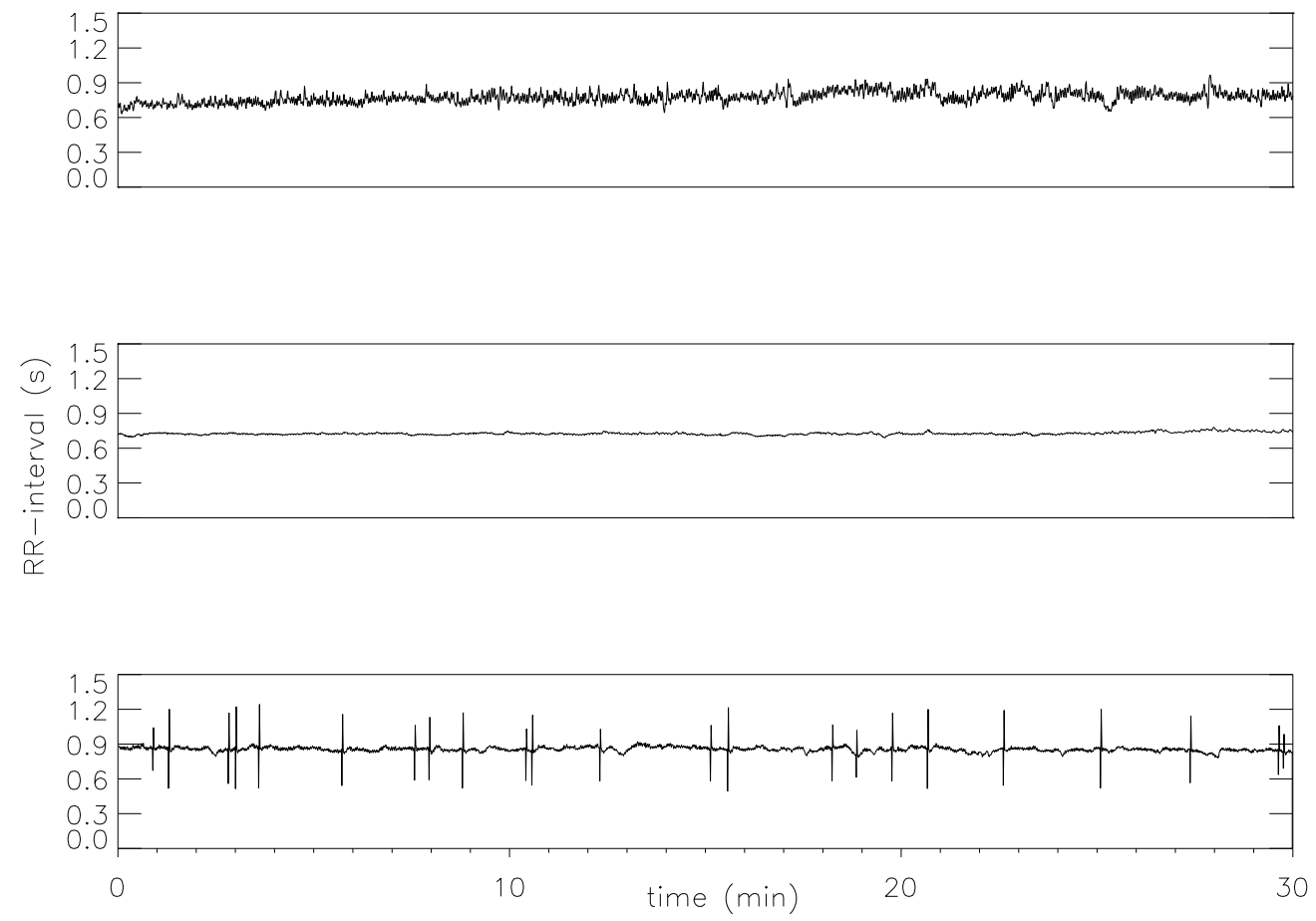

Figure 2: Tachograms of a healthy (top) and two ill persons

\section{Data and Pre-processing}

\section{$2.1 \quad$ Data}

The ECG recording has been done as follows: A 30 to 60 min 4 channel high resolution ECG (Frank leads and an additional diagonal lead) with a sampling frequency of $2000 \mathrm{~Hz}$ and 16 bit resolution (PC system with commercial available fast digitizing board) was obtained under rest condition. The Simson method [13] was used to calculate the sum vector magnitude from the three highly amplified (digital high pass filter, Butterworth characteristics $40 \mathrm{~Hz}$ ) leads X, Y and Z.

After digitizing and extracting of RR intervals by automatic procedures all RR time series have been checked by a technician and if necessary edited. The software RR detection algorithm is based on the cross correlation technique. 


\section{$2.2 \quad$ Patients}

In this preliminary study, we have included a sample of 43 patients subdivided in 3 groups. The first group consists of 21 healthy persons. In the second group there are 9 patients after myocardial infarction (MI) with low electrical risk (arrhytmias of low degree). Group 3 represents those 13 cardiac patients after MI for whom severe ventricular arrhytmias (sustained ventricular tachycardia) have been documented. Three examples of RR-interval-series are to seen in Fig. 2.

\subsection{Traditional Analysis}

In the time domain we have calculated the following standard parameters: the quotient of mean and standard deviation, the standard deviation of averages of NN intervals over 1 and over 5 minutes as well as the proportion of NN-interval-differences $>50$ and $>100 \mathrm{~ms}$ and the root mean square of successive differences.

From the estimated power spectrum we have determined the power of the 4 above mentioned frequency bands (ULF, VLF, LF, HF) and the ratios LF/whole power and LF/HF.

All these quantities that are basing on linear statistis are rather simple to calculate, but they do not lead to a satisfying detection of high risk patients. The rapid development in the theory of nonlinear dynamical systems has caused some optimism for a more appropriate understanding of such complex rhythms, as expressed in the HRV.

\section{Complexity Measures}

In the 80-ies it came up the wide-spread hope that many complicated systems observed in nature can be described by a few nonlinear coupled modes. The properties of these systems are characterized by fractal dimensions, Lyapunov exponents, or Kolmogorov-Sinai-entropy [2]. However, we now know that such a low dimensionality can be expected only for rather coherent phenomena, such as observed in laser systems. Physiological data, as studied here, seem to have a more complex structure, may be due to high-dimensional processes or due to the influence of random-like fluctuations. In this chapter, we present rather unconventional approaches to find some characteristics in these records. 


\subsection{Symbolic Dynamics}

Symbolic dynamics is based on a coarse-graining of the measurements, i.e. the data $t_{n}$ are transformed into a pattern whose elements are only a few symbols (letters from some alphabet). This way, the study of the dynamics simplifies to the description of symbol sequences. In doing so one loses some amount of detailed information, but some of the invariant, robust properties of the dynamics may be kept (Hao, 1991).

The first step is to find a suitable symbolic description. If we do not know a generating partition, there is no straightforward procedure for this problem, but it is context dependent (Kurths et al., 1994). Hence, we have to look for a coding procedure which is suitable for our purpose. From various tests we have found that for our purpose at least 4 different symbols are necessary. This leads to use two different kinds to transform the HRV records into symbol sequences. The first transformation refers to three given levels.

$$
s_{n}= \begin{cases}0, & \text { if } t_{n}>(1+a) \mu \\ 1 & , \text { if } t_{n}>\mu \text { and } t_{n} \leq(1+a) \mu \\ 2 & \text {, if } t_{n}>(1-a) \mu \text { and } t_{n} \leq \mu \\ 3, & \text { if } t_{n} \leq(1-a) \mu\end{cases}
$$

where $\mu$ denotes the mean RR-interval and $a$ is a special parameter specified in section 4 . The second transformation considers the kind of difference between two adjacent measurement values; it especially reflects dynamical properties of the record:

$$
\tilde{s}_{n}= \begin{cases}0, & \text { if } \triangle t_{n}>1.5 \sigma_{\triangle} \\ 1, & \text { if } \triangle t_{n}>0 \text { and } \triangle t_{n} \leq 1.5 \sigma_{\triangle} \\ 2, & \text { if } \triangle t_{n}>-1.5 \sigma_{\triangle} \text { and } \triangle t_{n} \leq 0 \\ 3 & \text {, if } \triangle t_{n} \leq-1.5 \sigma_{\triangle}\end{cases}
$$

with $\triangle t_{n}=t_{n+1}-t_{n}$ and $\sigma_{\triangle}$ is the variance of $\triangle t_{n}$. In the following we check, which of these transformations is more appropriate for our purpose.

Next, some classical parameters, which quantify different aspects of the behaviour of such a symbolic string $s_{n}$ are presented.

The first approach is to calculate the frequencies of occurring symbols. To investigate a rather broad range of dynamics, one should analyze long words. However, our data sets only contain 
about 2000 RR-intervals and the number of all possible words of length 1 basing on the alphabet, as introduced in eq. 1 , is $4^{l}$. We, therefore, count length-3 words as a good compromise between including some dynamics and the reliability in estimating the frequencies. With these frequencies one can distinguish rather uniform distributions from more complicated ones. This leads to the first measure of complexity which simply counts the number of forbidden words. For statistical reasons, we modify this idea somewhat and test for the number of words with a low probability of occurrence (probability less than 0.001).

A classical measure of symbol sequences is the Shannon entropy. From the probabilities $p\left(s^{k}\right)$ of words of length $k$ we get the Shannon entropy of $k^{\text {th }}$ order as follows

$$
H_{k}=-\sum_{s^{k}, p\left(s^{k}\right)>0} p\left(s^{k}\right) \log _{2} p\left(s^{k}\right) .
$$

This $H_{k}$ measures the average number of bits needed to specify an arbitrary word of length $\mathrm{k}$ in the symbolic string.

The concept of Renyi [12] entropy was introduced as a generalization of Shannon's ansatz

$$
H_{k}^{(q)}=(1-q)^{-1} \log _{2}\left(\sum_{s^{k}} p\left(s^{k}\right)^{q}\right)
$$

where $q$ is a real number and $q \neq 1$. It includes different averaging of probabilities. $H_{k}^{(q)}$ converges to Shannon entropy $H_{k}$ as $q \rightarrow 1$. Both, the Shannon entropy and the spectrum of Renyi entropies are measures of complexity which characterize systems as follows [17, 18]:

1. The complexity is zero for constant sequences.

2. In case of periodicity with prime period $m, m<k$ one gets $H=\log _{2} m$.

3. For uniform distributions it takes its maximum value $H=k \log _{2} \alpha$ where $\alpha$ is the number of symbols.

4. $H_{k}^{(q)}$ decreases with growing $q$.

5. If $q>1$ those words of length $k$ with large probability dominantly influence the Renyi entropy. This behaviour is strengthened for larger $q$ values. Vice versa, if $0<q<1$ then words with small probability mainly determine the value of $H_{k}^{(q)}$. 
In order to get reliable estimates of these $H_{n}$, which are also based on counting the frequencies of substrings, we calculate here entropy of order 3 only. A possible inhomogeneous structure inherent the data is checked by determining the Renyi entropies for $q=4$ and $q=0.25$.

It is important to note that all special complexity measures mentioned above do not include long-range correlations.

\subsection{Renormalized Entropy}

The main purpose of this paper is the comparison of the HRV of different persons to get some judgement of their risk for sudden cardiac death. As is well-known, the underlying system that generates the HRV is not closed, but an open one. From the viewpoint of general system theory, this means that different persons may have different mean energy. In such a case the immediate comparison of measures, such as Shannon entropy, may lead to some difficulties. Basing on a recent suggestion of Klimontovich [7], we, therefore, introduce here another complexity measure, the renormalized entropy. This approach, losely speaking, renormalizes the entropy obtained from a time series $x(t)$ of a certain person in such a manner that the mean effective energy coinsides with that of a reference person $x_{r}(t)$.

Starting from these two time series, we can easily estimate the corresponding probability distributions $f(z)$ and $f_{r}(z)$. By using formal arguments from thermodynamics the effective energy is defined as:

$$
h_{e f f}(z)=-\log f_{r}(z)
$$

The renormalization of $f_{r}$ into $\tilde{f}_{r}$ is constructed such that the mean effective energies $\left\langle h_{e f f}>\right.$ of $f$ and $\tilde{f}_{r}$ are equal. To make this idea operational, we first represent the distribution in terms of the canonical Gibbs distribution

$$
\tilde{f}_{r}(z)=e^{\frac{\Phi\left(T_{e f f}\right)-h_{e f f}(z)}{T_{e f f}}}
$$

which can be rewritten as

$$
\tilde{f}_{r}(z)=C\left(T_{e f f}\right) \cdot e^{-\frac{h_{e f f}(z)}{T_{e f f}}}
$$

where $T_{e f f}$ and $\Phi\left(T_{e f f}\right)$ are the effective temperature resp. the free effective energy. Because $h_{e f f}$ can be calculated from eq. (5), there are two unknowns in eq. (7): $C\left(T_{e f f}\right)$ and $T_{e f f}$. They are determined from the following two conditions. 


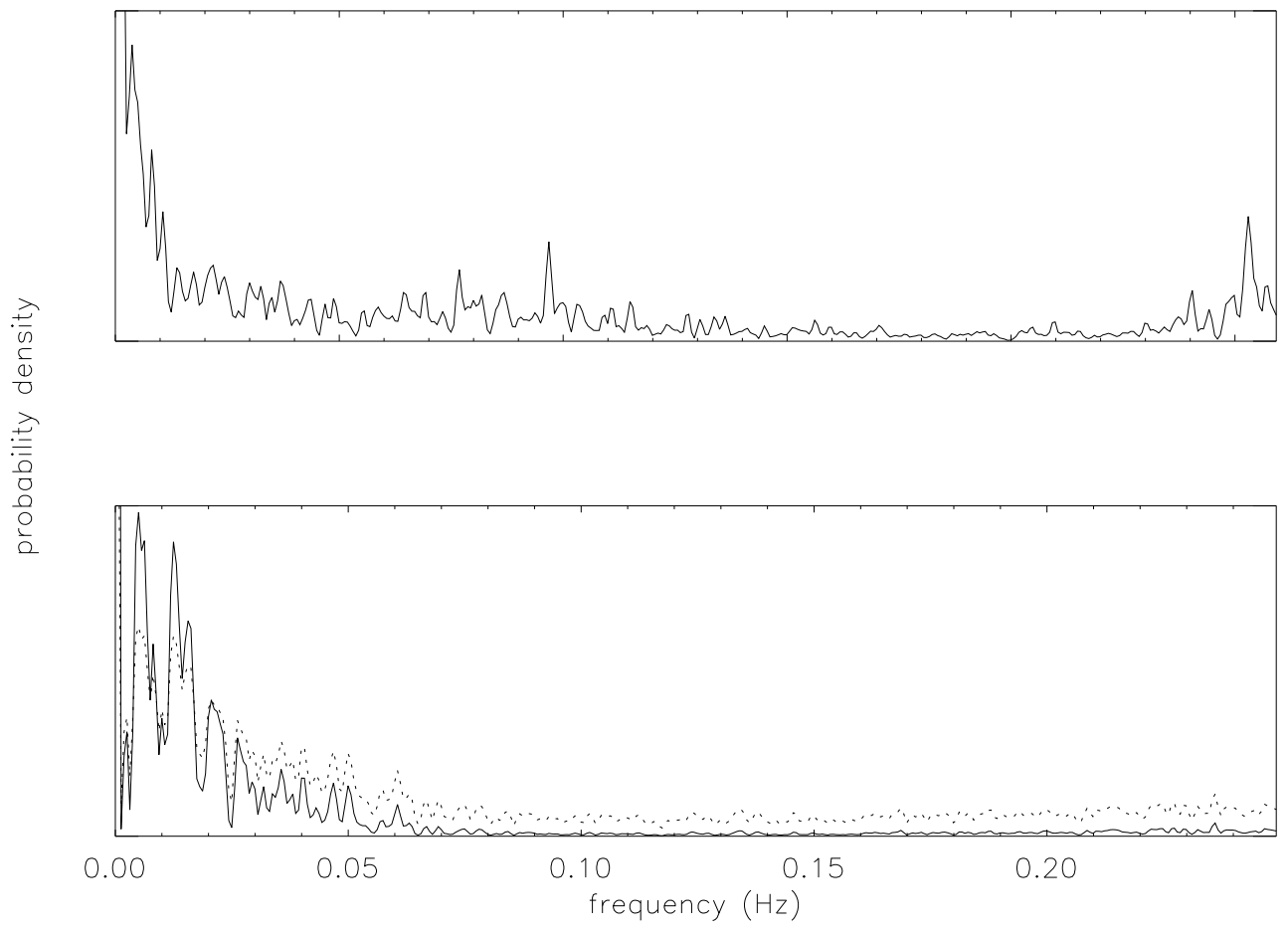

Figure 3: renormalization of distributions in the power spectrum: top - original distribution of the reference person, bottom - original (solid line) and renormalized (dashed) distribution of another person

a) normalization:

$$
\int \tilde{f}_{r}(z) d z=1
$$

b) equality of mean effective energy:

$$
\int h_{e f f}(z) \tilde{f}_{r}(z) d z=\int h_{e f f}(z) f(z) d z
$$

Hence, $\tilde{f}_{r}$ fulfils the properties wanted. Consequently, we can compare the Shannon entropies of $f$ and $\tilde{f}_{r}$

$$
H=-\int f(z) \log f(z) d z \quad \text { and } \quad \tilde{H}_{r}=-\int \tilde{f}_{r}(z) \log \tilde{f}_{r}(z) d x
$$

For that the renormalized entropy difference

$$
\triangle \tilde{H}=H-\tilde{H}_{r}
$$


is introduced. It is important to note that $\triangle \tilde{H}$ is a relative measure that depends on the reference person (system) chosen. Applying it to the logistic map, we have recently found that this renormalized entropy is the only complexity measure which clearly indicates all transitions between different regimes which are caused by this map (Saparin et al., 1994). Therefore, this new measure can also be a good tool to detect high risk patients.

\section{Results and Discussion}

We calculate all characteristics of the three main different approaches mentioned above from the HRV records described in section 2. The parameters in the time and in the frequency domain are determined as usually, i.e. 5 parameters in the time domain and 6 parameters in the frequency domain (as described in 2.3).

Next, we describe some details of the estimate of the complexity measures introduced in section 3. It comes out that the first transformation (eq. 1) into a symbol sequence is for our purpose more appropriate than the dynamical transformation (eq. 2). The optimum value of a in eq. 1 is about 0.1. For persons with cardiac risk, the distribution of length-3 words is concentrated on about 10 words (of 64 possible ones), whereas healthy persons are characterized by a more uniform distribution. An efficient criterion to distinguish both is then: persons have a risk if there are more than 44 words which seldom occur. As expected, the Shannon entropy is not so useful as the generalized Renyi entropies. Due to the higher variability of healthy persons, we expect that Renyi entropies for a rather small $q$ is much higher for this group than for the high risk group. The special criterion reads then: $H_{3}^{0.25}<3.6$ is an indication for cardiac risk. We also apply this kind of complexity measure for strings obtained from transformation eq. 2.

Our calculation of the renormalized entropy $\triangle \tilde{H}$ is based on the distribution of the trigonometric components, i.e. especially the power spectrum in the range $0-0.25 \mathrm{~Hz}$. We have tested several healthy probands as reference persons and have chosen that with the largest renormalized entropy. The corresponding power spectrum is shown in Fig. 3a. Note that this choice of a reference subject does not sensitively influence the results. Fig. $3 \mathrm{~b}$ demonstrates how the renormalization procedure influences a distribution. After choosing this reference person, the $\triangle \tilde{H}$ of all healthy persons under consideration is in the interval $(-0.75,0)$. Hence, an indication for cardiac risk is if $\triangle \tilde{H}$ is outside of this interval. We indeed find values in both directions; a very low $\triangle \tilde{H}$ expresses a strongly reduced 


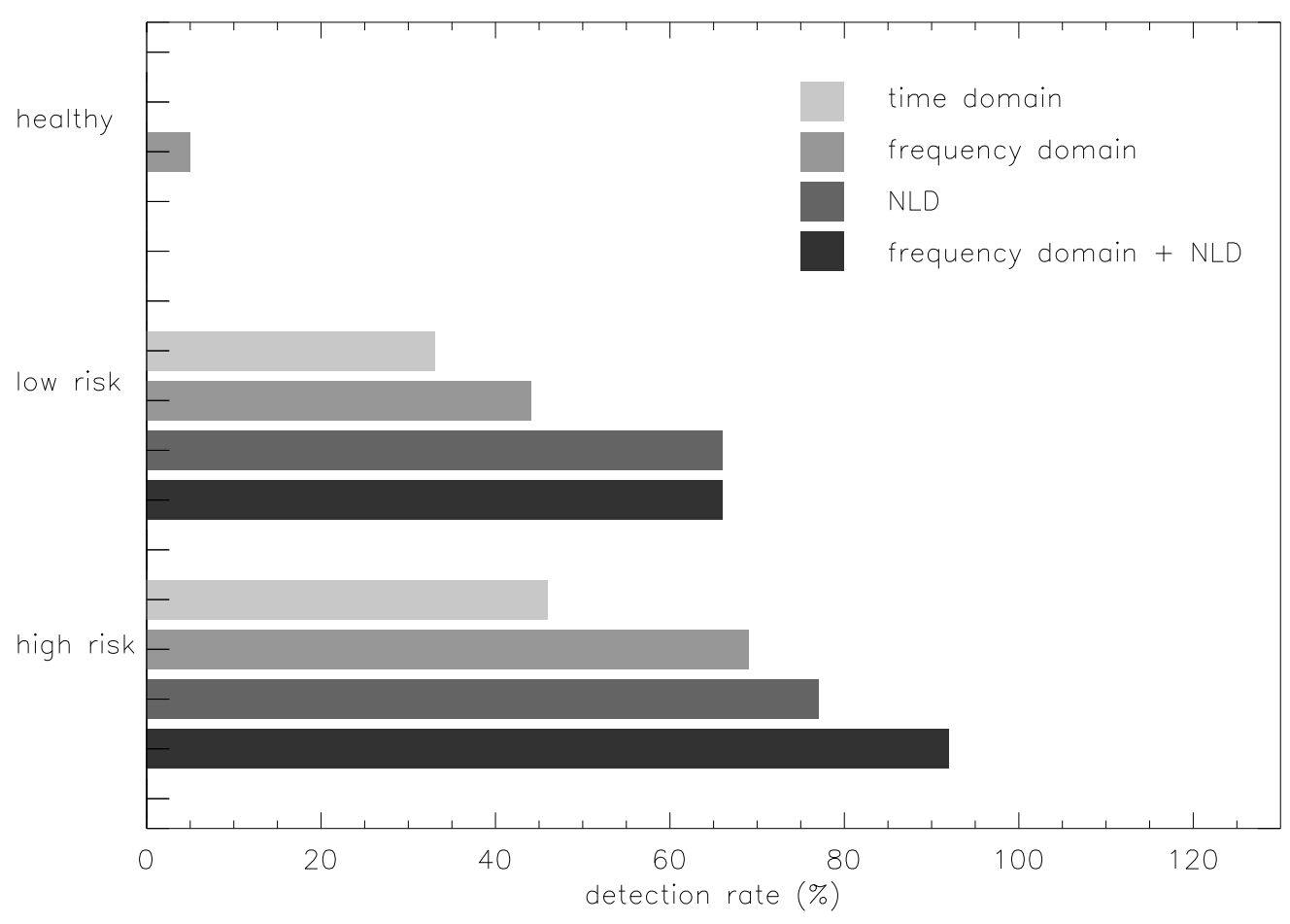

Figure 4: Comparison of the detection rate for high cardiac risk by means of different techniques. The subjects are subdivided in 3 groups classified by usual methods. NLD refers to comlexity measures obtained from nonlinear dynamics.

variability.

It is important to note that no healthy proband is misinterpreted by means of these complexity measures. To determine, on the other hand, the individual cardiac risk, it is more suitable if we consider an integrated risk that includes all 4 criteria discussed above. This is in accordance with the use of the special parameters in the time and frequency domain. Hence, we can compare three different risk estimates (Fig. 4):

a) As expected, the parameters in the time domain are less efficient than the other ones. By means of this risk, about $40 \%$ of the high risk patients are detected only. Therefore, this approach will not be further included.

b) The analysis in the frequency domain leads to a rather good distinction of the three groups. This seems to be due to the physiological meaning of some bands in the power spectrum. 
c) The risk basing on the complexity measures gives the best detection rate of the high risk patients.

Because the persons detected in the frequency domain and by complexity measures are not completely overlapping, we combine both. This way, a very good detection rate of high risk patients is obtained.

The evaluation of persons for whom only a low risk has so far been reported is an open problem. Here, we get an important difference between both kinds of tools. To check which techniques better fit to find high risk patients from this group, a more sophisticated medical characterization than the electric risk (LOWN4) is necessary..

\section{Summary}

We have applied the concept of complexity measures to determine the risk for sudden cardiac death from the HRV, a very actual clinical problem. By means of classical methods, especially parameters in the time domain, the individual risk cannot be defined precisely enough.

We have found some indications that two kinds of complexity measures are very promising:

a) Renyi entropies and the number of forbidden words which both are basing on the notion of symbolic dynamics as well as

b) a renormalized entropy which we have recently analyzed in the framework of complexity measures [14].

In combination with some parameters in the frequency domain, these quantities seem to define a rather precise definition of the individual risk. In contrast to this, the parameters in the time domain which are in broad use do not improve the detection rate.

It is important to note that one cannot find an optimum complexity measure. We guess that a combination of some such quantities which refers to different aspects, such as structural or dynamical properties, seems to be the most promising way. The complexity measure proposed by Pincus [11] as well as the criteria that are based on the description of long-range correlations (cf. Peng et al. [10]) should also be included to define the individual risk.

Finally, we would like to emphasize that our findings have to be validated by a larger and more representative number of patients, especially to check our optimized non-standard techniques. We 
also think that the study of the heart rhythms are in its infancy and should by continued by modeling the underlying processes and further analyses of measurements.

Table 1: Number of subjects found as risk by different techniques (chapter 2.3,3)

\begin{tabular}{|c|c|c|c||c|c|c|}
\hline group & $\begin{array}{c}\text { No. of subjects } \\
\text { subjects }\end{array}$ & $\begin{array}{c}\text { time } \\
\text { domain }\end{array}$ & $\begin{array}{c}\text { frequency } \\
\text { domain }\end{array}$ & $\begin{array}{c}\text { Renyi } \\
\text { information }\end{array}$ & $\begin{array}{c}\text { frequency } \\
\text { of words }\end{array}$ & $\Delta \tilde{S}$ \\
\hline healthy & 21 & 0 & 1 & 0 & 0 & 0 \\
\hline low risk & 9 & 3 & 4 & 6 & 5 & 6 \\
\hline high risk & 13 & 6 & 9 & 7 & 11 & 8 \\
\hline
\end{tabular}

\section{ACKNOWLEDGMENTS}

This research was supported in part by funding of the Ministerium für Wissenschaft, Forschung und Kultur des Landes Brandenburg. We are indebted to the unknown referees for their helpful remarks. 


\section{References}

[1] J. T. Bigger, J. L. Fleiss, L. M. Rolnitzky and R. C. Steinmann, "Stability over time of heart period variability in patients with previous myocardial infarction and ventricular arrhythmias", Am. J. Cardiol 69, 718-723 (1992).

[2] J. P. Eckmann and D. Ruelle, "Ergodic theory of chaos and strange attractors", Rev. Mod. Phys. 57, 617 (1985).

[3] A. L. Goldberger, "Fractal mechanisms", IEEE EMBS 11, 47-52 (1992).

"Is the normal heartbeat chaotic or homeostasis", New in physiological sciences 6, 87-91 (1991).

[4] B.-L. Hao, "Symbolic dynamics and characterization of complexity", Physica D 51, 611-176 $(1991)$.

[5] A. Hempelmann and J. Kurths, "Dynamics of the outburst series of SS Cygni", Astron. Astrophys. 232, 356-360 (1990).

[6] R. E. Kleiger, J. P. Miller, J. T. Bigger and A. J. Moss, "Decreased heart rate varibility and its association with increased mortality after acute myocardial infaction", Am. J. Cardiol. 59, $256-262(1987)$.

[7] Yu. L. Klimontovich, Turbulent motion and the Structure of Chaos (Kluwer Academic Publishers, Doddrecht, 1991).

[8] J. Kurths, A. Witt, H. Atmanspacher, F. Feudel, H. Scheingraber and R. Wackerbauer, General remarks on complexity. in Atmanspacher et al., Springer (1994).

[9] M. Malik, T. Farell and A. J. Camm, " Circadian rhythm of heart rate variability after acute myocardial infarction and its influence on the prognostic value of heart rate variability", $A m$. J. Cardiol 66, 1049-1054 (1990).

[10] C.-K. Peng, J. Mietus, J. m. Hausdorff, S. Havlin, H. E. Stanley and A. L. Goldberger, "Long-range correlations and non-Gaussian behaviour of the heartbeat", Phys. Rev. Lett. 70, 1343-1346 (1993). 
[11] S. Pincus (this issue).

[12] A. Renyi, Wahrscheinlichkeitsrechnung. Mit einem Anhang über Informationstheorie (Deutscher Verlag der Wissenschaften, Berlin, 1977).

[13] M. B. Simson, "Use of signals in the terminal QRS complex to identify patients with ventricular tachycardia after myocardial infarction", Circulation 64, 235-242 (1982).

[14] Saparin P., Witt A., Kurths J. and V. Anishenko, "The Renormalized Entropy - An Appropriate Complexity Measure". Chaos, Solitons and Fractals (in press)

[15] A. Voss, J. Kurths, H. Fiehring and H. J. Kleiner, "Frequenzanalyse hochverstaerkter EKGs mit Hilfe der Maximum-Entropie-Spektralschaetzung", Z. Klin. Med. 43, 1403-1406 (1988).

[16] A. Voss, J. Kurths and H. Fiehring, "Frequency domain analysis of the highly amplified ECG on basis of maximum entropy spectral estimation", Med. \& Biol. Eng. \& Comput. 30, 277-282 (1992).

[17] R. Wackerbauer, A. Witt, H. Atmanspacher, J. Kurths and H. Scheingraber, "Quantification of structural and dynamical complexity", Chaos, Solitons and Fractals 4, 133-173 (1994).

[18] A. Witt, J. Kurths, F. Krause and K. Fischer, "On the reversals of the Earth's magnetic field", GAFD, (1994, in press). 